\title{
Chronic Thromboembolic Pulmonary Hypertension Complicated by a Cavitating Lung Infection Caused by Mycobacterium intracellulare
}

\author{
Kenichi Okuda, Hirotoshi Matsui, Junko Suzuki, Nobuharu Ohshima, Kimihiko Masuda, \\ Akira Yamane, Atsuhisa Tamura, Hideaki Nagai, Shinobu Akagawa and Ken Ohta
}

\begin{abstract}
A 35-year-old man with a six-month history of progressive exertional dyspnea was referred to our institution. He had been diagnosed with Mycobacterium intracellulare pulmonary infection with lung cavitation two years earlier, and was being followed up without any medications. After being referred to our hospital, he underwent computed tomographic pulmonary angiography, which indicated a pulmonary thrombus and lung cavitation. Furthermore, right heart catheterization confirmed pulmonary hypertension, and we made a diagnosis of chronic thromboembolic pulmonary hypertension (CTEPH). Following successful pulmonary endarterectomy, the patient's symptoms and hemodynamics were significantly improved, with the disappearance of lung cavitation. It is important to suspect CTEPH in patients with unaccountable infectious lung cavities.
\end{abstract}

Key words: chronic thromboembolic pulmonary hypertension, Mycobacterium intracellulare, cavitation, pulmonary thromboembolism, pulmonary endarterectomy

(Intern Med 53: 1829-1833, 2014)

(DOI: 10.2169/internalmedicine.53.2252)

\section{Introduction}

Chronic thromboembolic pulmonary hypertension (CTEPH) is defined by an elevated mean pulmonary arterial pressure (mPAP) ( $\geq 25 \mathrm{mmHg}$ ) and a normal pulmonary capillary wedge pressure due to thromboembolic occlusion of the pulmonary arteries. Since nonspecific symptoms, such as progressive exertional dyspnea and coughing, are common in patients with CTEPH, CTEPH is rarely diagnosed at the initial visit. However, making an accurate and early diagnosis of CTEPH is important because certain treatments, including pulmonary endarterectomy (PEA), can significantly improve the clinical outcomes of affected patients. Although there are a few papers describing the presence of pulmonary cavitation associated with CTEPH, case reports of CTEPH complicated by nontuberculous mycobacterial infection have rarely been published. Therefore, we herein report the clinical findings and course of a patient with CTEPH complicated by a cavitating lung infection with Mycobacterium (M) intracellulare.

\section{Case Report}

A 35-year-old obese man was referred to our institution with a six-month history of gradually progressive dyspnea on exertion and a persistent cough. He had a past medical history of surgery of an appendico-cutaneous fistula two years prior to referral. At that time, he was incidentally diagnosed with $M$. intracellulare pulmonary infection based on a computed tomography (CT) scan of the chest, which revealed a cavity in the right lower lobe, and sputum cultures positive for $M$. intracellulare. Since then, he had been followed up without any medications because he presented with no symptoms. He had an unremarkable family history, with the exception of deep venous thrombosis in his cousin. He was a current smoker, with a history of 20 pack-years, and had been working in customer relations at a snack bar, which made him stand for more than fifteen hours a day. He denied illegal drug use and dust exposure in both his home 


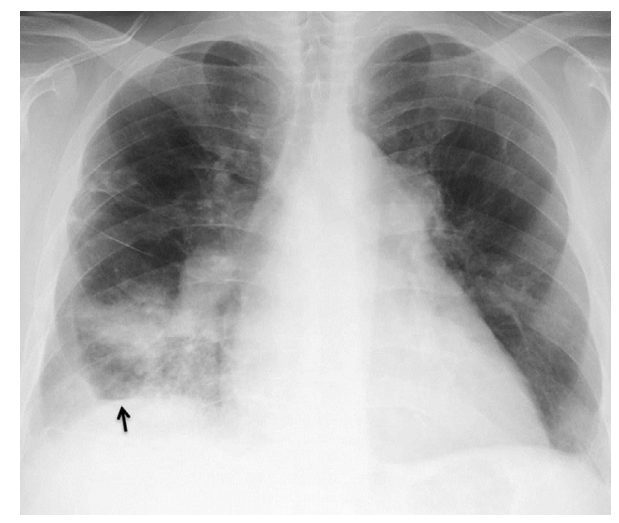

Figure 1. A chest radiograph showed an increase in the size of the cardiac shadow, bilateral hilar enlargement, and infiltrative shadows with cavitation (black arrow) in the right lower lung field.

and working environment.

Since sputum cultures obtained in the outpatient department were again positive for $M$. intracellulare, a standard regimen of oral antibiotics, i.e. rifampicin, ethambutol, and clarithromycin, was initiated. However, two weeks later, he developed intermittent bloody sputum with little improvement of the exertional dyspnea and was admitted to our hospital.

Up on admission, the patient complained of shortness of breath, even during mild activities, such as walking slowly or talking. He weighed $120 \mathrm{~kg}$ and was $177 \mathrm{~cm}$ in height. His vital signs were as follows: blood pressure, 143/55 $\mathrm{mmHg}$; pulse rate, regular at 109/min; respiratory rate, 24/ min; body temperature $36.2^{\circ} \mathrm{C}$. He was alert and with an oxygen saturation level of $95 \%$ while breathing room air. On auscultation, coarse crackles were heard in the right lower lung field during inspiration. He had regular heart sounds without murmurs, and pitting edema was noted in the lower extremities. The remainder of the physical examination was unremarkable.

The laboratory findings were as follows: hemoglobin,17.4 $\mathrm{g} / \mathrm{dL}$; white blood cell count, $6,200 / \mu \mathrm{L}$, with a normal differential cell count. Coagulation tests revealed an increased level of D-Dimer of $25.4 \mu \mathrm{g} / \mathrm{mL}$ and a decreased AT-III of 63.2\%. Although the levels of Protein $\mathrm{C}$ and $\mathrm{S}$ were decreased, the Protein $\mathrm{C}$ activity was within the normal limits. The C-reactive protein level was $1.62 \mathrm{mg} / \mathrm{dL}$, the lactate dehydrogenase level was $377 \mathrm{IU} / \mathrm{L}$ and the brain natriuretic peptide level was $588.7 \mathrm{pg} / \mathrm{mL}$. Both renal and liver function test results were normal. The findings of an arterial blood gas analysis performed on room air were consistent with respiratory alkalosis with hypoxia $\left(\mathrm{PaO}_{2}, 62.8 \mathrm{mmHg}\right.$; $\mathrm{PaCO}_{2}, 30.4 \mathrm{mmHg}$; $\mathrm{pH}, 7.496$ ). In addition, a microscopic examination of the patient's sputum smears revealed acidfast bacilli, and the cultures later yielded $M$. intracellulare.

An electrocardiogram showed sinus tachycardia and was otherwise normal. Pulmonary function tests were also within the normal limits. A transthoracic echocardiogram showed marked enlargement of the right ventricle and an increased tricuspid regurgitation peak gradient of $60 \mathrm{mmHg}$. Meanwhile, ultrasonography of the lower extremities disclosed small mural thrombi in the left popliteal vein. A chest radiograph showed an increase in the size of the cardiac shadow, with a cardiothoracic ratio of $56 \%$, in addition to bilateral hilar enlargement of the pulmonary arteries and infiltrative shadows with cavitation in the right lower lung field (Fig. 1). A CT scan of the chest demonstrated a thick-walled cavity and mass measuring 5 and $4.2 \mathrm{~cm}$ in diameter, respectively, with small nodular shadows scattered throughout the right lower lobe (Fig. 2A). In addition, an area of wedge-shaped subpleural consolidation, suggesting pulmonary infarction, was noted in the right middle lobe, as well as a mosaic perfusion pattern in the bilateral lung fields with right pleural effusion (Fig. 2B). Furthermore, CT pulmonary angiography revealed mural thrombi in the bilateral main pulmonary arteries (Fig. 2C, D). Although the left main pulmonary artery was partially embolized by thrombi, no abnormal findings were detected in the left lung field, except for a mosaic perfusion pattern.

The patient was diagnosed with pulmonary thromboembolism (PTE) based on the radiologic findings described above and, treated with anticoagulant therapy. Several days later, a ventilation/perfusion scan disclosed unmatched perfusion defects in the right middle and left upper lobes. In addition, right heart catheterization confirmed significant pulmonary hypertension (PH), with a mPAP of $60 \mathrm{mmHg}$, while the pulmonary capillary wedge pressure was normal at $6 \mathrm{mmHg}$. The values of pulmonary vascular resistance (PVR) and the cardiac index (CI) were 1,098 dyn.s.cm ${ }^{-5}$ and $1.69 \mathrm{~L} / \mathrm{min} / \mathrm{m}^{2}$, respectively. Furthermore, pulmonary angiography disclosed abrupt interruption in the right middle and lower pulmonary arteries in addition to complete obstruction in the left upper and lower pulmonary arteries (Fig. 3A). Finally, the patient was diagnosed with CTEPH complicated by pulmonary infection with $M$. intracellulare and cavitating lung lesions.

Subsequently, the patient was transferred to an expert center for PEA, a standard surgical procedure for CTEPH. He underwent successful PEA in which the organized thrombi in the pulmonary arteries were removed from the peripheral branches to the extent possible. After the surgery, both bosentan and sildenafil were administered together with anticoagulant therapy and the same antibiotics for M.intracellulare infection, except for rifampicin. One month after the operation, right heart catheterization revealed significant decreases in both the mPAP and PVR values to $39 \mathrm{mmHg}$ and 365 dyn.s.cm ${ }^{-5}$, respectively, while the CI was increased to $3.5 \mathrm{~L} / \mathrm{min} / \mathrm{m}^{2}$. Postoperative pulmonary angiography also demonstrated an improvement in the perfusion of the pulmonary arteries, which had been obstructed prior to PEA (Fig. 3B). Moreover, one year after the procedure, a chest CT scan showed the resolution of the cavity with a residual scar and a decreased number of the small nodular shadows in the right lower lobe (Fig. 4). Smears and cultures of the 

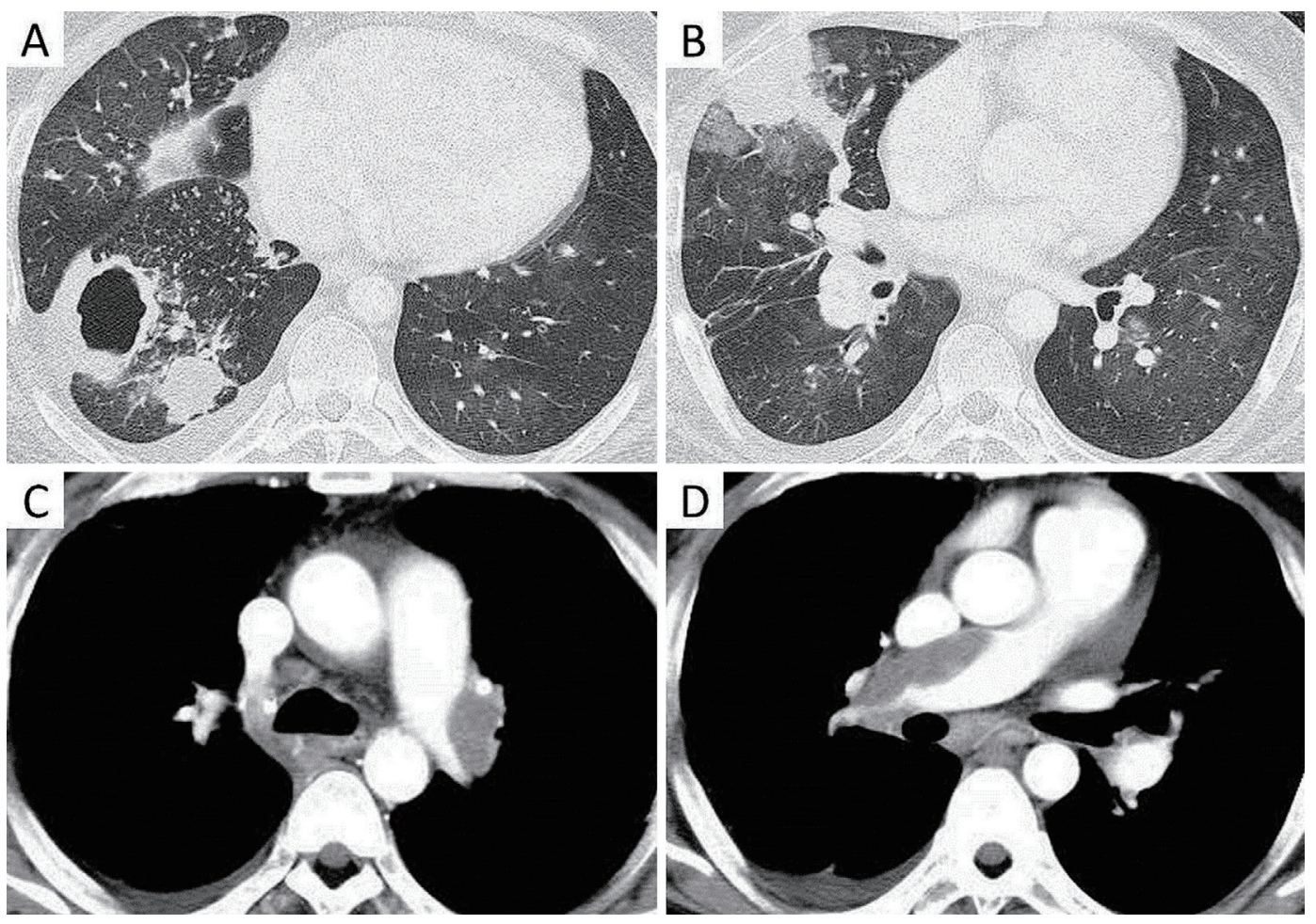

Figure 2. A CT scan of the chest demonstrate a thick-walled cavity and a mass with small nodular shadows in the right lower lobe (A). A wedge shaped subpleural consolidation is demonstrated in the right middle lobe as well as mosaic perfusion patterns in the bilateral lung fields (B). CT pulmonary angiography reveals mural thrombi in the bilateral main pulmonary arteries $(\mathrm{C}, \mathrm{D})$.
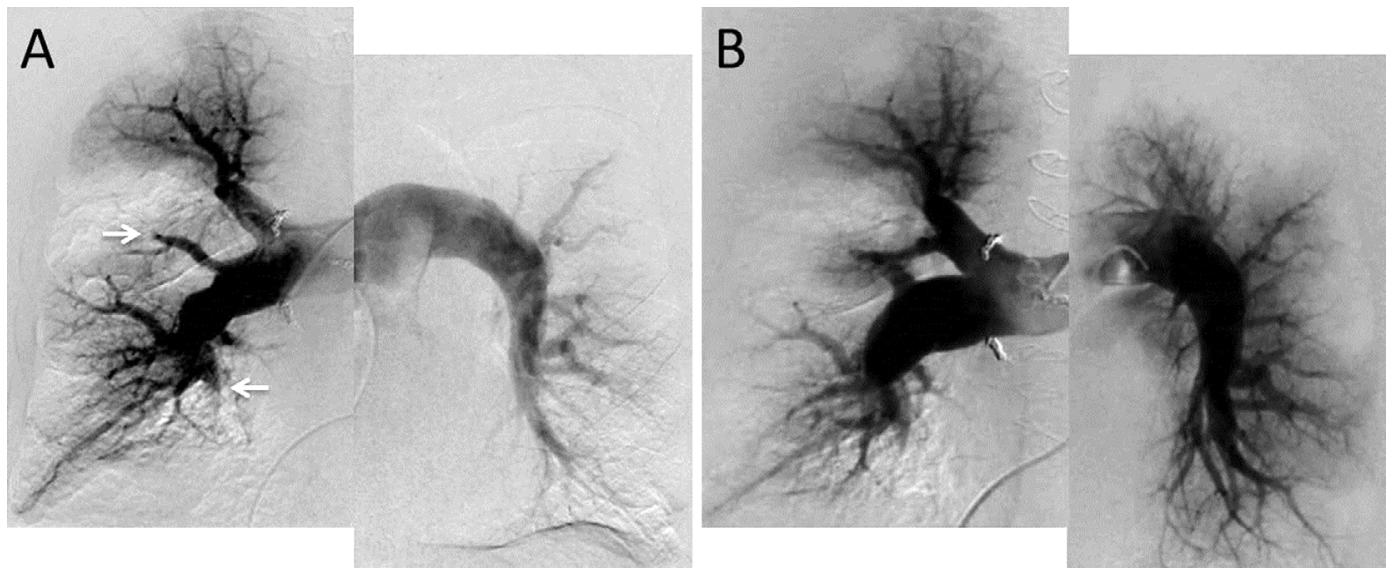

Figure 3. Preoperative pulmonary angiography shows the abrupt interruption in the right middle and lower pulmonary arteries (white arrows), complete obstruction in the left upper and lower pulmonary arteries (A). Postoperative pulmonary angiography demonstrates the improvement of perfusion in the bilateral pulmonary arteries (B).

patient's sputum subsequently became negative for acid-fast bacilli. In addition, his dyspnea on exertion significantly improved, thus allowing him to live his everyday life without oxygen therapy.

\section{Discussion}

Since the common symptoms of CTEPH, e.g. progressive exertional dyspnea, exercise intolerance, coughing and palpi- tations, are similar to those of other respiratory and cardiac diseases, many patients are not diagnosed with CTEPH at their first visit and do not receive appropriate treatment for CTEPH. Sato et al. reported that only 26 of $116 \mathrm{CTEPH}$ cases $(22 \%)$ were correctly diagnosed at the first visit (1). In the present case, CTEPH was not diagnosed for two years during the clinical course of pulmonary infection with $M$. intracellulare. CTEPH may have already been present two years earlier considering the detection of an enlarged main 


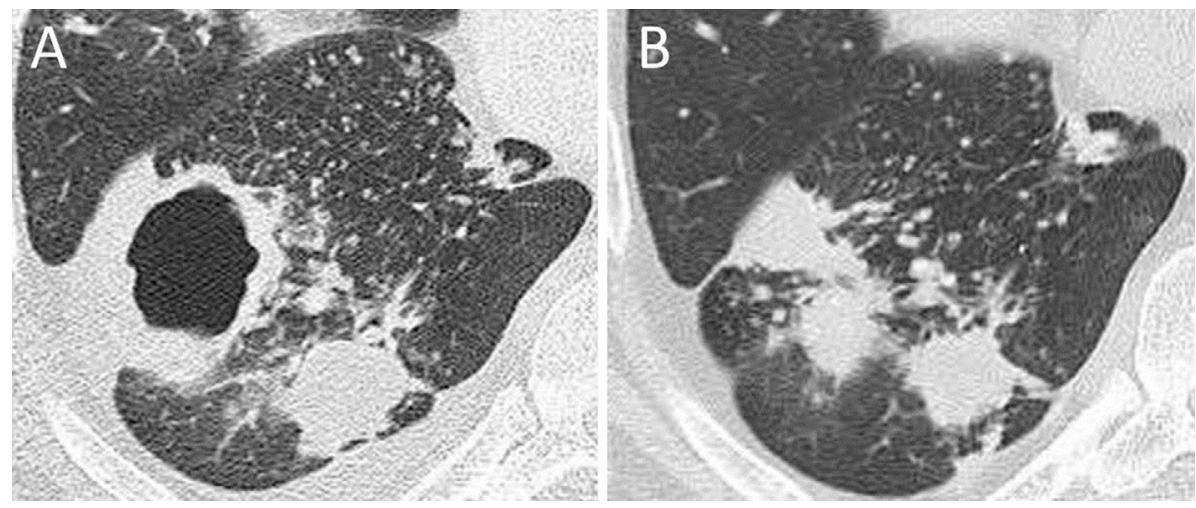

Figure 4. A CT scan of the chest, a year after PEA, shows resolution of the cavity with residual scaring and reduction of the small nodular shadows in the right lower lobe $(B)$ compared it before PEA (A).

pulmonary artery on a non-contrast CT scan of the chest, a findings that has been reported to be one of the most common radiological findings in patients with CTEPH (2).

The present patient exhibited cavitating lung lesions associated with CTEPH. Cavitation associated with PTE may result from aseptic necrosis of the infarcted lung tissue, secondary bacterial infection with subsequent abscess formation or the combination of both. However, the development of pulmonary cavitation after PTE is considered to be rare due to the dual blood supply of the lungs from both the bronchial and pulmonary arteries (3). Morgenthaler et al. reported that, in their study, lung infarction occurred in less than $15 \%$ of cases of acute PTE, of which only $5 \%$ were cavitated (4). Meanwhile, one large autopsy series including 550 patients with lung infarction revealed pulmonary cavitation in 23 cases (4.2\%) (5). Although reports of pulmonary cavities in CTEPH patients have rarely been published, Harris et al. reported that 11 of 104 patients (10.9\%) with CTEPH developed a cavity. The diagnosis of cavities in seven of these 11 patients followed the acute exacerbation of symptoms, including increased breathlessness, coughing and pyrexia, with the remaining cases being diagnosed during routine workups. In that report, three of the 11 cases involved clinical symptoms of infection, such as positive sputum cultures and an elevated C-reactive protein (CRP) level, while 10 of the 11 patients were treated with antibiotics, with clinical and radiological improvements. One case in which the cavity resolved spontaneously during follow-up has also been reported (6). Furthermore, Libby et al. documented both positive sputum cultures and elevated WBC counts in eight of 10 patients with lung infarction who developed pulmonary cavitation. In that report, organisms isolated from the sputum cultures included common Gramnegative rods, such as Escherichia coli, Pseudomonas aeruginosa and Proteus species (3). These reports suggest the possibility that not only an impaired circulation to the lung parenchyma, but also the bacterial infection itself, contribute to cavity formation in patients with PTE. The present case also indicates that both impaired perfusion and infection play important roles in the development of cavities in pa- tients with CTEPH, as the recovery of perfusion by PEA apparently facilitated the disappearance of the patient's Mycobacterium avium complex (MAC) cavity, which was treated with clarithromycin and ethambutol both before and after the surgery. We suspect that reperfusion aided the patient's tissue recovery and potentiated the antibiotic therapy.

In this case, the sputum cultures yielded $M$. intracellulare. We were able to find only two other Japanese patients with PTE complicated by MAC pulmonary disease $(7,8)$. The common clinical characteristics of these three cases included a high mPAP, cavitating lung lesions and positive sputum cultures for $M$. intracellulare. In all three cases, the areas of lung cavitation were located in the lower lobe, where perfusion was impaired due to the presense of pulmonary artery thrombi. On the other hand, Kurashima et al. reported that cavitation caused by MAC is frequently detected in the upper lobes (9). Moreover, the present patient did not exhibit any of the radiological features of a MAC cavity, i.e. a patent bronchus running into a cavitary legion on a CT scan (10), suggesting that the cavity observed in this case was unlikely to have been formed by simple MAC infection. Therefore, we consider that the unique distribution of MAC cavities noted in our and other reported cases is related to impaired perfusion induced by CTEPH.

The prognosis of patients with CTEPH remains very poor due to progressive $\mathrm{PH}$ and right heart failure. In one study, the 5-year survival rate of patients with CTEPH was 30\% when the mPAP was greater than $40 \mathrm{mmHg}$ and $10 \%$ when greater than $50 \mathrm{mmHg}(11)$. Surgery is the only potentially curative therapy for CTEPH, and PEA is the standard surgical procedure for removing pulmonary artery thrombi. As for long-term outcomes, Corsico et al. reported the 5-year survival rate in 157 patients with CTEPH who underwent PEA to be $84 \%$ (12). On the other hand, several reports have indicated that patients with a high preoperative PVR are more likely to exhibit postsurgical complications and a high mortality rate $(13,14)$. Therefore, performing immediate PEA after making an early and correct diagnosis is important in order to improve the prognosis in patients with CTEPH. 
We experienced a rare case of CTEPH complicated by $M$. intracellulare lung infection. We suggest that the presence of cavitating lung lesions associated with infection is one clue for the early diagnosis of CTEPH. Clinicians should consider $M$. intracellulare as a causative organism in cases of cavity formation among patients with CTEPH.

The authors state that they have no Conflict of Interest (COI).

\section{Acknowledgement}

The authors would like to express our gratitude to Professor Hitoshi Ogino and the staff of the Department of Cardiovascular Surgery, Tokyo Medical University Hospital for their participation in the successful operation for CTEPH in this patient.

\section{References}

1. Sato S, Sugiura T, Tanabe N, et al. A successful operative case of chronic thromboembolic pulmonary hypertension clinically diagnosed as bronchial asthma. Nihon Kokyuki Gakkai Zasshi (Annals of the Japanese Respiratory Society) 48: 836-841, 2010 (in Japanese, Abstract in English).

2. Hansell DM. Small-vessel diseases of the lung: CT-pathologic correlates. Radiology 225: 639-653, 2002.

3. Libby LS, King TE, LaForce FM, Schwarz MI. Pulmonary cavitation following pulmonary infarction. Medicine (Baltimore) 64: 342-348, 1985.

4. Morgenthaler TI, Ryu JH, Utz JP. Cavitary pulmonary infarct in immunocompromised hosts. Mayo Clin Proc 70: 66-68, 1995.

5. Levin L, Kernohan JW, Moersch HJ. Pulmonary abscess secondary to bland pulmonary infarction. Dis Chest 14: 218-232, 1948.

6. Harris H, Barraclough R, Davies C, et al. Cavitating lung lesions in chronic thromboembolic pulmonary hypertension. J Radiol Case Rep 2: 11-21, 2008.

7. Nishimura $\mathrm{C}$, Tsunoda $\mathrm{O}$, Lee $\mathrm{Y}$, et al. A case of pulmonary infection due to Mycobacterium intracellulare developed after pulmonary embolism. Kekkaku 60: 319-324, 1985 (in Japanese, Abstract in English).

8. Ota T, Tsutsuki K, Gomi E, et al. A case of thrombosis occuring in the main trunk of the pulmonary arterial trees with pulmonary infection due to Mycobacterium intracellulare. Nihon Kyobu Shikkan Gakkai Zasshi (The Japanese Journal of Thoracic Diseases) 25: 901-906, 1987 (in Japanese, Abstract in English).

9. Kurashima A, Horibe M. Distribution of pulmonary Mycobacterium avium complex (MAC) disease cavities and their course under chemotherapy. Kekkaku 87: 397-402, 2012 (in Japanese, Abstract in English).

10. Kim TS, Koh WJ, Han J, et al. Hypothesis on the evolution of cavitary lesions in nontuberculous mycobacterial pulmonary infection: thin-section CT and histopathologic correlation. Am J Roentgenol 184: 1247-1252, 2005.

11. Riedel M, Stanek V, Widimsky J, Prerovsky I. Longterm followup of patients with pulmonary thromboembolism. Late prognosis and evolution of hemodynamic and respiratory data. Chest 81: 151-158, 1982.

12. Corsico AG, D'Armini AM, Cerveri I, et al. Long-term outcome after pulmonary endarterectomy. Am J Respir Crit Care Med 178: 419-424, 2008.

13. Ishida $K$, Masuda $M$, Tanabe $N$, et al. Long-term outcome after pulmonary endarterectomy for chronic thromboembolic pulmonary hypertension. J Thorac Cardiovasc Surg 144: 321-326, 2012.

14. Mayer E, Jenkins D, Lindner J, et al. Surgical management and outcome of patients with chronic thromboembolic pulmonary hypertension: results from an international prospective registry. J Thorac Cardiovasc Surg 141: 702-710, 2011.

(C) 2014 The Japanese Society of Internal Medicine http://www.naika.or.jp/imonline/index.html 